\title{
Micromole per Liter per Kilogram per Meter Squared
}

National Cancer Institute

\section{Source}

National Cancer Institute. Micromole per Liter per Kilogram per Meter Squared. NCI

Thesaurus. Code C119529.

A unit of concentration equal to micromoles per liter, divided by kilograms per meter squared. 